\title{
On approximation of homeomorphisms of a Cantor set
}

\author{
by \\ Konstantin Medynets (Kharkov)
}

\begin{abstract}
We continue the study of topological properties of the group $\operatorname{Homeo}(X)$ of all homeomorphisms of a Cantor set $X$ with respect to the uniform topology $\tau$, which was started by Bezuglyi, Dooley, Kwiatkowski and Medynets. We prove that the set of periodic homeomorphisms is $\tau$-dense in $\operatorname{Homeo}(X)$ and deduce from this result that the topological group $(\operatorname{Homeo}(X), \tau)$ has the Rokhlin property, i.e., there exists a homeomorphism whose conjugacy class is $\tau$-dense in $\operatorname{Homeo}(X)$. We also show that for any homeomorphism $T$ the topological full group $[[T]]$ is $\tau$-dense in the full group $[T]$.
\end{abstract}

1. Introduction. Many famous problems in ergodic theory involve the use of topologies on the group $\operatorname{Aut}(X, \mathcal{B}, \mu)$ of all measure-preserving transformations of a standard measure space. The first results on group topologies of $\operatorname{Aut}(X, \mathcal{B}, \mu)$ appeared in [Hal 1]. Halmos introduced two topologies $d_{\mathrm{u}}$ and $d_{\mathrm{w}}$, which were called later the uniform and weak topologies, respectively. He defined the uniform topology $d_{\mathrm{u}}$ by saying that two automorphisms $T$ and $S$ are "close" to each other if the quantity $\mu(\{x \in X$ : $T x \neq S x\})$ is small enough. The weak topology is generated by the sets of the form $N(T ; E ; \varepsilon)=\{S \in \operatorname{Aut}(X, \mathcal{B}, \mu): \mu(S E \triangle T E)<\varepsilon\}$, where $T \in \operatorname{Aut}(X, \mathcal{B}, \mu)$ and $E \in \mathcal{B}$. The use of these topologies turned out to be very fruitful and led to many outstanding results in ergodic theory (for references, see, for example, $[\mathrm{B}-\mathrm{K}-\mathrm{M}]$ and $[\mathrm{C}-\mathrm{F}-\mathrm{S}])$. One of the most relevant results in the theory is the Rokhlin lemma [Ro] stating that the set of periodic automorphisms is $d_{\mathrm{u}}$-dense in $\operatorname{Aut}(X, \mathcal{B}, \mu)$.

The idea of investigation of transformation groups by means of introducing various topologies into these groups was used in [B-D-K 1] and [B-D-K 2], where the authors considered the groups $\operatorname{Aut}(X, \mathcal{B})$ of all automorphisms of a standard Borel space and the group $\operatorname{Homeo}(X)$ of all

2000 Mathematics Subject Classification: Primary 37B05; Secondary 54H11.

Key words and phrases: Borel automorphisms of a Cantor set, homeomorphism of a Cantor set, Rokhlin lemma, full group of a homeomorphism. 
homeomorphisms of a Cantor set $X$ with several topologies analogous to those in ergodic theory.

Following [B-D-K 2], we continue studying the group $\operatorname{Homeo}(X)$ of all homeomorphisms of a Cantor set $X$ with the topology $\tau$ (cf. Definition 1.1), which is obviously a direct analog of the topology $d_{\mathrm{u}}$. We show that the set of all periodic homeomorphisms is $\tau$-dense in $\operatorname{Homeo}(X)$ (Corollary 2.2). This result can be treated as a topological version of the Rokhlin lemma. As a corollary, we prove that the set of topologically free homeomorphisms is $\tau$-dense in $\operatorname{Homeo}(X)$ (Theorem 2.8). Recall that a homeomorphism is called topologically free if the set of aperiodic points is dense.

In [G-K], an interesting class of topological groups was defined: by definition, a topological group has the Rokhlin property if it has an element whose conjugacy class is dense. The authors raised the question which groups possess this property. At the moment, there is an extensive list of such groups, including the group $\operatorname{Homeo}(X)$ with the topology generated by the metric $D(T, S)=\sup _{x \in X} d(T x, S x)$, where $d$ is a metric on $X$ compatible with the topology $[\mathrm{Gl}-\mathrm{W}]$, and the group $\operatorname{Aut}(X, \mathcal{B}, \mu)$ with the weak topology [Hal 2]. See also the paper [Ke-Ros] for a general approach to the study of groups with dense conjugacy classes.

Motivated by this, we present a uniform approach allowing us to show that the topological groups $(\operatorname{Aut}(X, \mathcal{B}), \tau)$ and $(\operatorname{Homeo}(X), \tau)$ have the Rokhlin property (Theorem 2.5).

Furthermore, inspired by the remarkable work [G-P-S], where full groups were indispensable in the study of orbit equivalence of Cantor minimal systems, we study the full groups $[T]$ with their dense subsets for arbitrary homeomorphisms $T \in \operatorname{Homeo}(X)$.

In this context, we show that for any $T \in \operatorname{Homeo}(X)$, the topological full group $[[T]]$ is $\tau$-dense in the full group $[T]$ (Theorem 2.1).

In the last section, we give a description of homeomorphisms from the topological full group $[[T]]$ for $T$ aperiodic (Theorem 3.3). We consider a subgroup $\Gamma_{Y}$ of $[[T]]$, which is an increasing union of permutation groups, and find a criterion for $\Gamma_{Y}$ to be $\tau$-dense in $[[T]]$ (Theorem 3.4).

Background. Throughout the paper, $X$ denotes a Cantor set and $\mathcal{B}$ stands for the $\sigma$-algebra of Borel subsets of $X$. A one-to-one Borel map $T$ of $X$ onto itself is called an automorphism of $(X, \mathcal{B})$. Denote by $\operatorname{Aut}(X, \mathcal{B})$ the group of all automorphisms of $(X, \mathcal{B})$ and by $\operatorname{Homeo}(X)$ the group of all homeomorphisms of $X$.

Following $[\mathrm{B}-\mathrm{K}]$, recall the definition of the uniform topology on $\operatorname{Aut}(X, \mathcal{B})$. Let $\mathcal{M}_{1}(X)$ denote the set of all Borel probability measures on $X$. For $T, S \in \operatorname{Aut}(X, \mathcal{B})$, define $E(T, S)=\{x \in X: T x \neq S x\}$. 
Definition 1.1. The uniform topology $\tau$ on $\operatorname{Aut}(X, \mathcal{B})$ is defined by the base of neighborhoods $\mathcal{U}=\left\{U\left(T ; \mu_{1}, \ldots, \mu_{n} ; \varepsilon\right)\right\}$, where

$$
U\left(T ; \mu_{1}, \ldots, \mu_{n} ; \varepsilon\right)=\left\{S \in \operatorname{Aut}(X, \mathcal{B}): \mu_{i}(E(S, T))<\varepsilon, i=1, \ldots, n\right\} .
$$

Here $T \in \operatorname{Aut}(X, \mathcal{B}), \mu_{1}, \ldots, \mu_{n} \in \mathcal{M}_{1}(X)$, and $\varepsilon>0$.

As $\operatorname{Homeo}(X)$ is a subgroup of $\operatorname{Aut}(X, \mathcal{B})$, we also denote by $\tau$ the topology on $\operatorname{Homeo}(X)$ induced from $(\operatorname{Aut}(X, \mathcal{B}), \tau)$.

Observe that $\operatorname{Aut}(X, \mathcal{B})$ and $\operatorname{Homeo}(X)$ are Hausdorff topological groups with respect to the uniform topology $\tau$. More results on topological properties of $\operatorname{Aut}(X, \mathcal{B})$ and $\operatorname{Homeo}(X)$ with respect to $\tau$ can be found in $[\mathrm{B}-\mathrm{D}-\mathrm{K} 1$, B-D-K 2, B-D-M, B-K-M, B-M].

Let $T \in \operatorname{Aut}(X, \mathcal{B})$. A point $x \in X$ is called periodic of period $n>0$ if $T^{n} x=x$ and $T^{i} x \neq x$ for $i=1, \ldots, n-1$. If $T^{n} x \neq x$ for $n \neq 0$, the point $x$ is called aperiodic. We say that $T$ is aperiodic if it has no periodic points. Note that for any $T \in \operatorname{Aut}(X, \mathcal{B})$ the set $X$ can be decomposed into a disjoint union of Borel sets $X=X_{\infty} \cup \cup_{n>1} X_{n}$, where $X_{n}$ consists of all points of period $n$ and $X_{\infty}$ is formed by all aperiodic points. Notice that some $X_{n}$ 's may be empty. Moreover, for every $X_{n}$ with $n<\infty$ there exists a Borel set $X_{n}^{0} \subset X_{n}$ such that $X_{n}=\bigcup_{i=0}^{n-1} T^{i} X_{n}^{0}$ is a disjoint union. We call $\left\{X_{\infty}, X_{1}, X_{2}, \ldots\right\}$ the canonical partition of $X$ associated to $T$.

Recall that a finite family of disjoint Borel sets $\xi=\left\{A, T A, \ldots, T^{n-1} A\right\}$ is called a $T$-tower with base $B(\xi)=A$ and height $h(\xi)=n$. A partition $\Xi=\left\{\xi_{1}, \xi_{2}, \ldots\right\}$ of $X$ is called a Kakutani-Rokhlin $(K-R)$ partition if every $\xi_{i}$ is a $T$-tower. For a $\mathrm{K}-\mathrm{R}$ partition $\Xi$, we denote $\bigcup_{n>1} B\left(\xi_{i}\right)$ by $B(\Xi)$ and call it the base of the K-R partition. Notice that $T^{-1} B(\Xi)=\bigcup_{\xi \in \Xi} T^{h(\xi)-1} B(\xi)$.

For $T \in \operatorname{Aut}(X, \mathcal{B})$, let $\operatorname{Orb}_{T}(x)=\left\{T^{n} x: n \in \mathbb{Z}\right\}$ denote the $T$-orbit of $x$. To any $T \in \operatorname{Homeo}(X)$, we can assign two full groups $[T]_{\mathrm{C}}$ and $[T]_{\mathrm{B}}$, where

$$
\begin{aligned}
& {[T]_{\mathrm{C}}=\left\{S \in \operatorname{Homeo}(X): \operatorname{Orb}_{S}(x) \subseteq \operatorname{Orb}_{T}(x), x \in X\right\}} \\
& {[T]_{\mathrm{B}}=\left\{S \in \operatorname{Aut}(X, \mathcal{B}): \operatorname{Orb}_{S}(x) \subseteq \operatorname{Orb}_{T}(x), x \in X\right\} .}
\end{aligned}
$$

Here the subscripts $\mathrm{C}$ and $\mathrm{B}$ indicate the Cantor and Borel dynamics, respectively. Clearly, $[T]_{\mathrm{C}}$ is a subgroup of $[T]_{\mathrm{B}}$. Observe that if $S \in[T]_{\mathrm{B}}$, then there is a Borel function $n_{S}: X \rightarrow \mathbb{Z}$ such that $S x=T^{n_{S}(x)} x$ for all $x \in X$. The subgroup $[[T]]=\left\{S \in[T]_{\mathrm{C}}: n_{S}\right.$ is continuous $\}$ is called the topological full group of $T$.

One of the main results in the approximation theory of Borel automorphisms is a Borel version of the Rokhlin lemma. The following $\tau$-version of the Rokhlin lemma was proved in [B-D-K 1, Proposition 3.6]. We also refer the reader to [N, Section 7] and [W, Section 4] for measure-free versions of the result. 
TheOREM 1.2. Let $T$ be an aperiodic automorphism of $X$. Then there exists a sequence of periodic automorphisms $P_{n} \in \operatorname{Aut}(X, \mathcal{B})$ such that $P_{n} \stackrel{\tau}{\rightarrow} T$ as $n \rightarrow \infty$. Moreover, the $P_{n}$ can be taken from $[T]_{\mathrm{B}}$.

Denote by $\mathrm{Per}_{0}$ the set of all homeomorphisms $P$ such that $P^{n}=\mathbb{I}$ for some $n \in \mathbb{N}$; and for $T \in \operatorname{Homeo}(X)$, set $\operatorname{Per}_{0}(T)=\operatorname{Per}_{0} \cap[[T]]$.

2. Rokhlin lemma. In this section, we prove a topological version of the Rokhlin lemma, namely, we show that the set of periodic homeomorphisms is $\tau$-dense in $\operatorname{Homeo}(X)$. Then we deduce several corollaries of this result. In particular, we prove that the topological group $(\operatorname{Homeo}(X), \tau)$ has the Rokhlin property and the topological full group $[[T]]$ is $\tau$-dense in $[T]_{\mathrm{B}}$ for any $T \in \operatorname{Homeo}(X)$.

THEOREM 2.1.

(1) The set $\mathcal{P} e r_{0}$ is $\tau$-dense in $\operatorname{Homeo}(X)$.

(2) Let $T \in \operatorname{Homeo}(X)$. Then for any automorphism $S \in[T]_{\mathrm{B}}$ and any $\tau$-neighborhood $U=U\left(S ; \mu_{1}, \ldots, \mu_{p} ; \varepsilon\right)$ of $S$ there exists a periodic homeomorphism $P \in[[T]]$ such that $P \in U$.

Proof. Notice that statement (1) is an immediate corollary of (2). By Theorem 1.2, it is enough to prove (2) for $S$ periodic.

Let us sketch the main stages of the proof:

(i) We find a finite number of disjoint $S$-towers consisting of closed sets and "almost" covering the entire space $X$ with respect to the measures $\mu_{i}$ such that on each level of these $S$-towers the automorphism $S$ coincides with a power of $T$.

(ii) We extend the $S$-towers found in (i) to clopen ones constructed by means of powers of $T$.

(iii) Using the clopen towers, we define a periodic homeomorphism $P$ which belongs to $U\left(S ; \mu_{1}, \ldots, \mu_{p} ; \varepsilon\right)$.

(i) Let $\Xi=\left\{X_{1}, X_{2}, \ldots\right\}$ be the canonical Borel partition of $X$ associated to $S$. Without loss of generality, we will assume that the sets $X_{i}$ are nonempty for all $i \in \mathbb{N}$.

We first find $N \in \mathbb{N}$ such that

$$
\mu_{j}\left(X_{1} \cup \cdots \cup X_{N}\right)>1-\varepsilon / 3 \quad \text { for all } j=1, \ldots, p .
$$

For $n \geq 1$, set $Z_{n}=\left\{x \in X: S x=T^{i} x\right.$ for some $\left.-n \leq i \leq n\right\}$. For $i \geq 1$ define

$$
X_{i}^{0}(n)=\bigcap_{j=0}^{i-1} S^{-j}\left(S^{j} X_{i}^{0} \cap Z_{n}\right),
$$


where $X_{i}=X_{i}^{0} \cup S X_{i}^{0} \cup \cdots \cup S^{i-1} X_{i}^{0}$ is a disjoint union. Since $S \in[T]_{\mathrm{B}}$, we have $X_{i}=\bigcup_{n \geq 1} X_{i}(n)$, where $X_{i}(n)=\bigcup_{j=0}^{i-1} S^{j} X_{i}^{0}(n)$. Then find $K \in \mathbb{N}$ such that

$$
\mu_{j}\left(\bigcup_{i=1}^{N}\left(X_{i} \backslash X_{i}(K)\right)\right)<\varepsilon / 3 \quad \text { for all } j=1, \ldots, p .
$$

Denote by $\mathcal{S}_{i}$ the set of all maps from $\{0, \ldots, i-1\}$ to $\{-K, \ldots, K\}$. For $\sigma \in \mathcal{S}_{i}$, set

$$
X_{i}^{0}(K, \sigma)=\bigcap_{j=0}^{i-1} S^{-j}\left(\left\{x \in S^{j} X_{i}^{0}(K): S x=T^{\sigma(j)} x\right\}\right) .
$$

Thus, we get a finite cover $X_{i}^{0}(K)=\bigcup_{\sigma \in \mathcal{S}_{i}} X_{i}^{0}(K, \sigma)$. Applying the standard argument, make the $X_{i}^{0}(K, \sigma)$ 's disjoint and denote the resulting sets by $X_{i}^{0}(K, \sigma)$ again. Some of the $X_{i}^{0}(K, \sigma)$ 's may be empty, but, without loss of generality, we will assume they are not. Observe that $S$ restricted to $S^{j} X_{i}^{0}(K, \sigma)$ is equal to $T^{\sigma(j)}$ for $i \geq 1, j=0, \ldots, i-1$, and $\sigma \in \mathcal{S}_{i}$. This means that $S$ is a homeomorphism on $S^{j} X_{i}^{0}(K, \sigma)$.

For every $X_{i}(K, \sigma)=\bigcup_{j=0}^{i-1} S^{j} X_{i}^{0}(K, \sigma)$, find a closed set $A_{i}^{0}(\sigma) \subset X_{i}^{0}(K, \sigma)$ such that

$$
\mu_{j}\left(\bigcup_{i=1}^{N} \bigcup_{\sigma \in \mathcal{S}_{i}}\left(X_{i}(K, \sigma) \backslash A_{i}(\sigma)\right)\right)<\varepsilon / 3 \quad \text { for } j=1, \ldots, p,
$$

where $A_{i}(\sigma)=\bigcup_{j=0}^{i-1} S^{j} A_{i}^{0}(\sigma)$.

(ii) Summing up the above, we see that $\left\{A_{i}(\sigma): 1 \leq i \leq N, \sigma \in \mathcal{S}_{i}\right\}$ is a family of disjoint closed $S$-towers such that $S$ restricted to $S^{j} A_{i}^{0}(\sigma)$ is equal to $T^{\sigma(j)}$. Furthermore, it follows from $(2.1)-(2.3)$ that

$$
\mu_{j}\left(\bigcup_{i=1}^{N} \bigcup_{\sigma \in \mathcal{S}_{i}} A_{i}(\sigma)\right)>1-\varepsilon
$$

As the closed $S$-towers $A_{i}(\sigma)$ are disjoint, we can find clopen sets $\bar{A}_{i}^{0}(\sigma) \supset$ $A_{i}^{0}(\sigma)$ so that all the sets $\bar{A}_{i}^{0}(\sigma)$ and $T^{\sigma(0)+\cdots+\sigma(j)} \bar{A}_{i}^{0}(\sigma)$ are mutually disjoint for $i=1, \ldots, N, j=0, \ldots, i-2$, and $\sigma \in \mathcal{S}_{i}$.

(iii) Define the periodic homeomorphism $P$ as follows:

$$
P x=\left\{\begin{array}{llc}
T^{\sigma(0)} x & \text { if } x \in \bar{A}_{i}^{0}, \\
T^{\sigma(j+1)} x & \text { if } x \in T^{\sigma(0)+\cdots+\sigma(j)} \bar{A}_{i}^{0}(\sigma), & \sigma \in \mathcal{S}_{i}, \\
T^{-\sigma(0)-\cdots-\sigma(i-2)} x & \text { if } x \in T^{\sigma(0)+\cdots+\sigma(i-2)} \bar{A}_{i}^{0}(\sigma), & 0 \leq j \leq i-3, \\
x & \text { otherwise. }
\end{array}\right.
$$


Clearly, $P$ is well-defined and belongs to $[[T]]$. By the definition of $P$,

$$
\{x \in X: P x=S x\} \supset \bigcup_{i=1}^{N} \bigcup_{\sigma \in \mathcal{S}_{i}} A_{i}(\sigma) .
$$

Hence, (2.4) shows that $P \in U\left(S ; \mu_{1}, \ldots, \mu_{p} ; \varepsilon\right)$, completing the proof.

Remark. After this work was submitted, B. Miller showed how using ideas of the proof above one can generalize Theorem 2.1(2) to any countable group acting by homeomorphisms on a zero-dimensional Polish space [Mil].

Rokhlin property. We give several immediate corollaries of Theorem 2.1, which have the well-known analogs in ergodic theory.

Corollary 2.2. Let $T \in \operatorname{Homeo}(X)$. Then, for every $\tau$-neighborhood $U$ of $T$, there exists a homeomorphism $P \in \operatorname{Per}_{0}(T) \cap U$ whose associated canonical partition is clopen.

The next statement generalizes Theorem 4.5 of [B-K] proved originally for minimal homeomorphisms.

Corollary 2.3. Let $T \in \operatorname{Homeo}(X)$. The topological full group $[[T]]$ of $T$ is $\tau$-dense in $[T]_{\mathrm{C}}$.

As $\operatorname{Homeo}(X)$ is not $\tau$-closed in $\operatorname{Aut}(X, \mathcal{B})$, in [B-D-K 2] the authors brought up the question of describing the closure of $[[T]]$ in $(\operatorname{Aut}(X, \mathcal{B}), \tau)$. They answered it for minimal homeomorphisms (see Theorem 2.8 of [B-D-K 2]) and we generalize it to an arbitrary homeomorphism.

Corollary 2.4. Let $T \in \operatorname{Homeo}(X)$. Then ${\overline{[[T]]^{\tau}}}^{\tau}=\overline{[T]}_{\mathrm{C}}^{\tau}=[T]_{\mathrm{B}}$.

Definition. A topological group $G$ has the Rokhlin property if the action of $G$ on itself by conjugation is topologically transitive, i.e. there is an element of $G$ whose conjugacy class is dense.

The following proposition extends the list of topological groups that have the Rokhlin property. See also [Gl-W] and [Ke-Ros] for other examples.

Theorem 2.5. The topological groups $(\operatorname{Aut}(X, \mathcal{B}), \tau)$ and $(\operatorname{Homeo}(X), \tau)$ have the Rokhlin property.

Proof. We prove the theorem for $(\operatorname{Homeo}(X), \tau)$ only, the other case being similar.

Take a decomposition $X=\left\{x_{0}\right\} \cup \bigcup_{i \geq 1} X_{i}$ such that the $X_{i}$ 's are nonempty clopen sets with $\operatorname{diam}\left(X_{i} \cup\left\{x_{0}\right\}\right) \rightarrow 0$ as $i \rightarrow \infty$. Let $S$ be a homeomorphism such that $S x_{0}=x_{0}$ and $S^{i} x=x, S^{j} x \neq x$ for any $x \in X_{i}, j=1, \ldots, i-1$. Our goal is to show that we can approximate any $T \in \operatorname{Homeo}(X)$ by elements from the conjugacy class of $S$. By Corollary 2.2, it suffices to approximate periodic homeomorphisms whose canonical partitions are clopen. So, suppose $T$ has a clopen partition $X=\bigcup_{i=1}^{k} Y_{i}$, where 
$Y_{i}$ is the set of all points having $T$-period $n_{i}$ for some $n_{i} \geq 0$. Observe that there exists a clopen set $Y_{i}^{0}$ such that $Y_{i}=\bigcup_{j=0}^{n_{i}-1} T^{j} Y_{i}^{0}$ is a disjoint union (see Lemma 3.2 of [B-D-K 2]). Analogously, there exists a clopen set $X_{n_{i}}^{0}$ with $X_{n_{i}}=\bigcup_{j=0}^{n_{i}-1} S^{j} X_{n_{i}}^{0}$ being a disjoint union.

Let $U=U\left(T ; \mu_{1}, \ldots, \mu_{p} ; \varepsilon\right)$ be a $\tau$-neighborhood of $T$. Take a non-empty clopen $T$-invariant set $Z$ with $\mu_{i}(Z)<\varepsilon$ for $i=1, \ldots, p$. Without loss of generality, we may assume that $Y_{i}^{0} \backslash Z$ is not empty for $i=1, \ldots, k$. Let $R_{i}$ be any homeomorphism from $X_{n_{i}}^{0}$ onto $Y_{i}^{0} \backslash Z$. Define a homeomorphism $R$ as follows: let $R(x)$ be equal to $T^{j} R_{i} S^{-j}(x)$ whenever $x \in S^{j} X_{n_{i}}^{0}$ for $i=1, \ldots, k, j=0, \ldots, n_{i}-1$ and let $R$ map the rest of $X$ onto $Z$. It is not hard to check that $R S R^{-1} \in U$.

In the setting of Borel dynamics, we need to produce a periodic transformation that has uncountably many orbits of any finite length. Then, the application of the Rokhlin lemma shows that its conjugacy class is dense.

REMARK. Let $p$ be the topology on $\operatorname{Homeo}(X)$ generated by the metric $D(T, S)=\sup _{x \in X} d(T x, S x)$, where $d$ is a metric on $X$ compatible with the topology. In $[\mathrm{Gl}-\mathrm{W}]$, it is shown that $(\operatorname{Homeo}(X), p)$ has the Rokhlin property. Moreover, the elements whose conjugacy classes are dense form a residual set with respect to $p$.

Topologically free homeomorphisms. It is interesting to compare the topological properties of the set Ap of all aperiodic homeomorphisms with respect to both topologies $\tau$ and $p$. The following statement is proved in [B-D-K 2, Theorem 2.1].

Theorem 2.6. The set Ap is dense in $(\operatorname{Homeo}(X), p)$.

However, the situation in $(\operatorname{Homeo}(X), \tau)$ is completely different. The set Ap is nowhere dense with respect to $\tau$. To see this, one can check that Ap is $\tau$-closed in Homeo $(X)$. Then an application of Theorem 2.1 implies the result.

The question we investigate in this section is: "How can we extend the class of aperiodic homeomorphisms to produce a $\tau$-dense class?". Apparently, the most natural extension of aperiodic homeomorphisms is the class of topologically free homeomorphisms.

Definition. A homeomorphism is topologically free if the set of all aperiodic points is dense.

In Theorem 2.8, we prove that the set of topologically free homeomorphisms is $\tau$-dense. To begin with, we need the following lemma on homeomorphism extensions proved in $[\mathrm{Kn}-\mathrm{R}]$. We will need the arguments used in its proof. Thus, we give a sketch of the proof, but without going into the details. 
LEMma 2.7. Let $A$ and $B$ be closed nowhere dense subsets of Cantor sets $X$ and $Y$, respectively. Suppose there is a homeomorphism $h: A \rightarrow B$. Then $h$ can be extended to a homeomorphism $h^{*}: X \rightarrow Y$ such that $\left.h^{*}\right|_{A}=h$.

Sketch of proof. Find clopen sets $U_{i}$ and $V_{j}$ such that $X \backslash A=\bigsqcup_{i>1} U_{i}$, $Y \backslash B=\bigsqcup_{j \geq 1} V_{j}$, and their diameters tend to zero. Find points $a_{i} \in A$ such that $\operatorname{dist}\left(U_{i}, A\right)=\operatorname{dist}\left(U_{i}, a_{i}\right)$ and $b_{j} \in B$ with $\operatorname{dist}\left(V_{j}, B\right)=\operatorname{dist}\left(V_{j}, b_{j}\right)$.

Set $I=J=\mathbb{N}$. There exist injective functions $f: I \rightarrow J$ and $g: J \rightarrow I$ such that

$$
\begin{array}{ll}
\operatorname{dist}\left(U_{i}, a_{i}\right)>\operatorname{dist}\left(V_{f(i)}, h\left(a_{i}\right)\right) & \text { for } i \in I, \\
\operatorname{dist}\left(V_{j}, b_{j}\right)>\operatorname{dist}\left(U_{g(j)}, h^{-1}\left(b_{j}\right)\right) & \text { for } j \in J .
\end{array}
$$

Applying the usual Schröder-Bernstein argument to $f$ and $g$, find disjoint partitions $I=I^{\prime} \sqcup I^{\prime \prime}$ and $J=J^{\prime} \sqcup J^{\prime \prime}$ such that $f\left(I^{\prime}\right)=J^{\prime}$ and $g\left(J^{\prime \prime}\right)=I^{\prime \prime}$.

Let $\phi$ be an arbitrary homeomorphism of $U^{\prime}=\bigcup_{i \in I^{\prime}} U_{i}$ onto $V^{\prime}=$ $\bigcup_{j \in J^{\prime}} V_{i}$ such that $\phi\left(U_{i}\right)=V_{f(i)}$. Analogously, let $\psi$ be a homeomorphism of $V^{\prime \prime}=\bigcup_{j \in J^{\prime \prime}} V_{j}$ onto $U^{\prime \prime}=\bigcup_{i \in I^{\prime \prime}} U_{i}$ such that $\psi\left(V_{j}\right)=U_{g(j)}$.

Define

$$
h^{*}(x)= \begin{cases}\phi(x), & x \in U^{\prime}, \\ \psi^{-1}(x), & x \in U^{\prime \prime}, \\ h(x), & x \in A .\end{cases}
$$

For the verification of continuity of $h^{*}$, we refer the reader to $[\mathrm{Kn}-\mathrm{R}]$.

THEOREM 2.8. The set of topologically free homeomorphisms is $\tau$-dense in $\operatorname{Homeo}(X)$.

Proof. By Corollary 2.2, it suffices to approximate the homeomorphisms from Per $_{0}$ that have clopen canonical partitions. Assume that $R$ belongs to $\mathrm{Per}_{0}$ and its canonical partition $X=X_{n_{1}} \cup \cdots \cup X_{n_{m}}$ is clopen. Recall that $X_{n_{i}}$ consists of all points with period $n_{i}$. Consider a $\tau$-neighborhood $U=U\left(R ; \mu_{1}, \ldots, \mu_{k} ; \varepsilon\right)$. Since the $X_{n_{i}}$ 's are $R$-invariant and clopen, we will prove the theorem under the assumption that $X=X_{n_{i}}$ for some $i$ and leave the generalization to the reader.

Suppose $X=\bigcup_{i=0}^{p-1} R^{i} F$ is a clopen partition and $R^{p} x=x$ for all $x \in X$. Using the standard Cantor argument, find a closed nowhere dense set $P \subset R^{p-1} F$ such that $\mu_{i}(P)>1-\varepsilon$ for $i=1, \ldots, k$. Repeating the proof of Lemma 2.7, we extend the homeomorphism $R: P \rightarrow R P$ to a homeomorphism $T: R^{p-1} F \rightarrow F$ so that the homeomorphism $T^{*} \in \operatorname{Homeo}(X)$ defined as $\left.T^{*}\right|_{R^{p-1} F}=\left.T\right|_{R^{p-1} F}$ and $T^{*}=P$ elsewhere is topologically free. To do this, it suffices to choose the functions $\psi$ and $\phi$ so that $\phi(x) \neq R x$ and $\psi^{-1}(x) \neq R x$ for $x \in R^{p-1} F \backslash P$. Since $E\left(T^{*}, R\right)=R^{p-1} F \backslash P$, we conclude that $T^{*} \in U$. 


\section{Structure of homeomorphisms from a topological full group.}

In this section, we discuss the structure of homeomorphisms from the topological full group $[[T]]$ for any aperiodic $T \in \operatorname{Homeo}(X)$.

Consider a Cantor aperiodic system $(X, T)$. A Borel set $Y \subset X$ is called wandering if $T^{n} Y \cap Y=\emptyset$ for all $n \geq 1$.

Definition. We say that a closed wandering set $Y$ is basic if every clopen neighborhood of $Y$ meets every $T$-orbit.

THEOREM 3.1. Every Cantor aperiodic system has a basic set.

Sketch of proof. Applying the argument developed in [B-D-M, Theorem 2], we can find a decreasing sequence $\left\{U_{n}\right\}$ of clopen sets such that: $U_{n+1} \subset U_{n} ; T^{i} U_{n} \cap U_{n}=\emptyset$ for $i=1, \ldots, n-1$; and $U_{n}$ meets every $T$-orbit. Then $Y=\bigcap_{n} U_{n}$ is a basic set.

REMARK. For more results on basic sets and their interaction with Bratteli diagrams, see $[\mathrm{M}]$.

Fix a triple $(X, T, Y)$, where $(X, T)$ is a Cantor aperiodic system and $Y$ is a basic set. Consider a clopen neighborhood $U$ of $Y$. It is not hard to check that for every $x \in U$, there is $n=n(x)>0$ such that $T^{n} x \in U$. Therefore, it follows from the definition of a basic set that, by applying the first return function, we can construct a clopen K-R partition $\Xi$ of $X$ with base $B(\Xi)=U$.

Take a decreasing sequence $\left\{U_{n}\right\}$ of clopen sets such that $Y=\bigcap_{n} U_{n}$. Constructing clopen K-R partitions for the $U_{n}$ 's and refining them, we prove the following:

TheOREm 3.2. Let $(X, T, Y)$ be a Cantor aperiodic system with a basic set $Y$. There exists a sequence $\left\{\mathcal{P}_{n}\right\}$ of clopen $K-R$ partitions of $X$ such that for all $n \geq 1$ :

(i) $\mathcal{P}_{n+1}$ refines $\mathcal{P}_{n}$;

(ii) $h_{n+1}>h_{n}$, where $h_{n}$ is the height of the lowest T-tower in $\mathcal{P}_{n}$;

(iii) $B\left(\mathcal{P}_{n}\right) \supset B\left(\mathcal{P}_{n+1}\right)$;

(iv) the sequence $\left\{\mathcal{P}_{n}\right\}$ generates the clopen topology of $X$;

(v) $\bigcap_{n} B\left(\mathcal{P}_{n}\right)=Y$.

We will follow here the method developed in $[\mathrm{B}-\mathrm{K}]$ for minimal homeomorphisms (see also $[\mathrm{K}-\mathrm{W}]$ ). Let $\mathcal{P}$ be a clopen $\mathrm{K}-\mathrm{R}$ partition with towers $\mathcal{P}(i), i=1, \ldots, k$. We will define two partitions $\alpha=\alpha(\mathcal{P})$ and $\alpha^{\prime}=\alpha^{\prime}(\mathcal{P})$ of $\{1, \ldots, k\}$. We say that $J$ is an atom of $\alpha$ if there exists a subset $J^{\prime}$ (then said to belong to $\alpha^{\prime}$ ) such that

$$
T\left(\bigcup_{i \in J} T^{h(i)-1} D_{i}\right)=\bigcup_{i^{\prime} \in J^{\prime}} D_{i^{\prime}}
$$


and for every proper subset $J_{0}$ of $J$, the $T$-image of $\bigcup_{i \in J_{0}} T^{h(i)-1} D_{i}$ is not a union of atoms from $\mathcal{P}$. It follows from (3.1) that $J^{\prime}$ is uniquely defined by $J$ and $T$.

Let $S \in[[T]]$. Then there are a finite set $K \subset \mathbb{Z}$ and a clopen partition $\mathcal{E}=\left\{E_{k}: k \in K\right\}$ of $X$ such that $S x=T^{k} x$ for $x \in E_{k}$ and $k \in K$. Denote by $\mathcal{E}(K)$ the clopen partition $\left\{S^{k} E_{k}: k \in K\right\}$. By Theorem 3.2, find a K-R partition $\mathcal{P}=\{\mathcal{P}(i): i=1, \ldots, k\}$ with $\mathcal{P}(i)=\left\{D_{0, i}, \ldots, D_{h(i)-1, i}\right\}$ and $D_{j+1, i}=T D_{j, i}$ that refines $\mathcal{E}$ and $\mathcal{E}(K)$ and so that $K \subset(-h, h)$, where $h$ is the height of the lowest $T$-tower in $\mathcal{P}$.

Let $\mathcal{F}=\{(j, i): i=1, \ldots, k, j=0, \ldots, h(i)-1\}$. Observe that for every pair $(j, i) \in \mathcal{F}$ there is a unique $l=l(j, i) \in K$ such that

$$
S\left(D_{j, i}\right)=T^{l} D_{j, i}
$$

Divide $\mathcal{F}=\mathcal{F}(\mathcal{P})$ into three disjoint sets $\mathcal{F}_{\text {in }}, \mathcal{F}_{\text {top }}$ and $\mathcal{F}_{\text {bot }}$ as follows:

(a) $(j, i) \in \mathcal{F}_{\text {in }}$ if $S\left(D_{j, i}\right) \subset \mathcal{P}(i)$, i.e. $0 \leq l+j \leq h(i)-1$;

(b) $(j, i) \in \mathcal{F}_{\text {top }}$ if $S\left(D_{j, i}\right)$ goes through the top of $\mathcal{P}(i)$, i.e. $l+j \geq h(i)$;

(c) $(j, i) \in \mathcal{F}_{\text {bot }}$ if $S\left(D_{j, i}\right)$ goes through the bottom of $\mathcal{P}(i)$, i.e. $l+j<0$; here $l$ is taken from (3.2).

Let $\alpha$ and $\alpha^{\prime}$ be the partitions of $\{1, \ldots, k\}$ defined by $T$ and $\mathcal{P}$. For $J \subset\{1, \ldots, k\}$, set $h_{J}=\min \{h(i): i \in J\}$. For $J \in \alpha$ and $J^{\prime} \in \alpha^{\prime}$, let

$$
F_{1}(r, J)=\bigcup_{i \in J} D_{h(i)-h_{J}+r, i}, \quad F_{2}\left(r^{\prime}, J^{\prime}\right)=\bigcup_{i^{\prime} \in J^{\prime}} D_{r, i^{\prime}},
$$

where $r=0, \ldots, h_{J}-1$ and $r^{\prime}=0, \ldots, h_{J^{\prime}}-1$.

Definition. We say that $S \in[[T]]$ belongs to $\Gamma(\mathcal{P})$ if for each pair $(j, i) \in \mathcal{F}$ the following conditions hold:

(a) if $(j, i) \in \mathcal{F}_{\text {top }}$ and $D_{j, i} \subset E_{l}$, then $F_{1}\left(h_{J}-h(i)+j, J\right) \subset E_{l}$, where $J$ is the atom of $\alpha$ containing $i$;

(b) if $(j, i) \in \mathcal{F}_{\text {bot }}$ and $D_{j, i} \subset E_{l}$, then $F_{2}\left(j, J^{\prime}\right) \subset E_{l}$, where $J^{\prime}$ is the atom of $\alpha^{\prime}$ containing $i$.

Condition (a) means that whenever the set $D_{j, i}$ goes through the top of $\mathcal{P}(i)$ under the action of $S$, then the entire level $F_{1}(r, J)$ containing $D_{j, i}$ also goes through the top of $\mathcal{P}$. Similarly, one can explain condition (b) by taking the $D_{j, i}$ 's and levels $F_{2}\left(j, J^{\prime}\right)$ containing them that go through the bottom of $\mathcal{P}$. Observe that if $(j, i) \in \mathcal{F}_{\text {in }}$, then the entire levels $F_{1}(r, J)$ and $F_{2}\left(j, J^{\prime}\right)$ containing $D_{j, i}$ remain "within" $\mathcal{P}$.

Clearly, $\Gamma(\mathcal{P})$ is a finite set. The following theorem reveals the structure of homeomorphisms from $[[T]]$ for an arbitrary aperiodic homeomorphism $T$. Notice that this structure was found earlier for minimal homeomorphisms (see Theorem 2.2 in $[\mathrm{B}-\mathrm{K}]$ ). Since our proof is similar to that in [B-K, Theorem 2.2], we omit it. 
TheOREm 3.3. Let $(X, T, Y)$ be a Cantor aperiodic system with a basic set $Y$ and a sequence $\left\{\mathcal{P}_{n}\right\}$ of $K-R$ partitions satisfying the conditions of Theorem 3.2. Then $[[T]]=\bigcup_{n} \Gamma\left(\mathcal{P}_{n}\right)$ with $\Gamma\left(\mathcal{P}_{n}\right) \subset \Gamma\left(\mathcal{P}_{n+1}\right)$.

The subgroup $\Gamma_{Y}$. Let $(X, T)$ be a Cantor aperiodic system with a basic set $Y$. Define the subgroup $\Gamma_{Y}$ of $[[T]]$ as follows: $S \in \Gamma_{Y}$ if $S \in \Gamma\left(\mathcal{P}_{n}\right)$ (and hence $S \in \Gamma\left(\mathcal{P}_{m}\right)$ for $\left.m>n\right)$ implies that $\mathcal{F}\left(\mathcal{P}_{n}\right)=\mathcal{F}_{\text {in }}$. In other words, $S \in \Gamma_{Y}$ if no level from $\mathcal{P}_{n}$ goes over the top or through the bottom under the action of $S$. This means that $S$ acts as a permutation on each $T$-tower from $\mathcal{P}_{n}$. Therefore, the group $\Gamma_{Y}$ is an increasing union of permutation groups.

Our objective is to find a criterion for $\Gamma_{Y}$ to be dense in $[T]$.

Remark. (1) Denote by $[[T]]_{Y}$ the subgroup of $[[T]]$ consisting of the homeomorphisms that preserve the forward $T$-orbit of every $y \in Y$, i.e., $S \in[[T]]_{Y}$ if $S\left(\left\{T^{n} y: n \geq 0\right\}\right)=\left\{T^{n} y: n \geq 0\right\}$. Observe that $\Gamma_{Y} \subset[[T]]_{Y}$.

(2) The subgroup $[[T]]_{Y}$ is not $\tau$-dense in $[T]$. Therefore, so is $\Gamma_{Y}$. Indeed, take any $z \in T^{-1} Y$ and the Dirac measure $\delta_{z}$. Consider $S \in U:=$ $U\left(T ; \delta_{z} ; 1 / 2\right)$. As $z \notin Y$ and $S z=T z \in Y, S$ does not preserve the forward $T$-orbit of $T z$. Therefore, $U$ contains no elements from $[[T]]_{Y}$.

The fact that $\Gamma_{Y}$ is not $\tau$-dense in $[[T]]$ is mainly caused by the presence of discrete measures. We can partly overcome this obstacle by considering only continuous measures in the definition of the topology $\tau$. Denote by $\tau_{0}$ the topology defined by continuous measures as in Definition 1.1. One can check that $\tau_{0}$ is a Hausdorff group topology on $\operatorname{Homeo}(X)$. The next theorem answers the question when $\Gamma_{Y}$ is $\tau_{0}$-dense in $[T]$.

Theorem 3.4. Suppose we have a Cantor aperiodic system $(X, T)$ with a basic set $Y$. Then the subgroup $\Gamma_{Y}$ is $\tau_{0}$-dense in $[T]$ if and only if the basic set $Y$ is at most countable.

Proof. (1) Assume that $Y$ is uncountable. Take any continuous measure $\mu$ supported by $T^{-1} Y$. Then for every $S \in U:=U(T ; \mu ; 1 / 2)$ there is at least one $z \in T^{-1} Y$ such that $S z=T z$. This implies that $\left\{T^{n}(T z)\right.$ : $n \geq 0\}$ is not $S$-invariant. Therefore, by (1) of the remark above we conclude that $\Gamma_{Y} \cap U=\emptyset$.

(2) Now, assume that $Y$ is countable. Observe that by Corollary 2.3, it is enough to approximate homeomorphisms from $[[T]]$ with elements of $\Gamma_{Y}$. Consider $R \in[[T]]$ and a $\tau_{0}$-neighborhood $U=U\left(R ; \mu_{1}, \ldots, \mu_{p} ; \varepsilon\right)$ of $R$. By definition of $R$, the sets $E_{k}=\left\{x \in X: R x=T^{k} x\right\}, k \in K,|K|<\infty$, form a clopen partition of $X$. Let $k_{0}=\sup \{|k|: k \in K\}$. As $Y$ is countable, $\mu\left(T^{n} Y\right)=0$ for any continuous measure $\mu$ and integer $n$. Therefore, by Theorem 3.2 we can find a K-R partition $\mathcal{P}_{n}$ refining $\left\{E_{k}\right\}_{k \in K}$ such that 
$2 k_{0}<h_{n}$, where $h_{n}$ is the height of the lowest $T$-tower in $\mathcal{P}_{n}$, and

$$
\mu_{j}\left(\bigcup_{i=-k_{0}}^{k_{0}} T^{i} B\left(\mathcal{P}_{n}\right)\right)<\varepsilon \quad \text { for } j=1, \ldots, p .
$$

Define a homeomorphism $S \in \Gamma_{Y} \cap U$ as follows. Take a $T$-tower (say $\lambda=$ $\left.\left\{D, \ldots, T^{h(\lambda)-1} D\right\}\right)$ from $\mathcal{P}_{n}$. Consider an atom $T^{l} D$ of $\lambda$. We have two possibilities:

(i) The $R$-orbit of the set $T^{l} D$ does not leave the $T$-tower $\lambda$. In this case, we define $S$ to be equal to $R$ on the $R$-orbit of $T^{l} D$.

(ii) The set $T^{l} D$ leaves $\lambda$ under the action of $R$. Then there exist integers $q<0<d$ such that $R^{d+1} T^{l} D$ and $R^{q-1} T^{l} D$ do not lie in $\lambda$ entirely, whereas the sets $R^{j} T^{l} D, j=q, \ldots, d$, are contained in $\lambda$. In this case, we set $S=R$ on $R^{i} T^{l} D, i=q, \ldots, d-1$, and $S=R^{-d+q}$ on $R^{d} T^{l} D$.

Observe that the choice of $\mathcal{P}_{n}$ guarantees that

$$
R^{d} T^{l} D \subset \bigcup_{i=0}^{k_{0}} T^{i} D \cup \bigcup_{i=h(\lambda)-1-k_{0}}^{h(\lambda)-1} T^{i} D .
$$

Clearly, the homeomorphism $S$ constructed in (i) and (ii) is periodic, but it is not yet defined on the entire space. To extend its domain, we consider an atom $T^{w} D$ of $\lambda$ on which $S$ is not defined yet, and repeat (i) and (ii) with $T^{w} D$.

Repeating this procedure with every atom of $\lambda$, we define $S$ on $\lambda$. Moreover, $\lambda$ is $S$-invariant. By construction, $S$ coincides with $P$ everywhere, except maybe on the set $\bigcup_{i=0}^{k_{0}} T^{i} D \cup \bigcup_{i=h(\lambda)-1-k_{0}}^{h(\lambda)-1} T^{i} D$.

To finish constructing $S$, we need to repeat the argument for every $T$ tower of $\mathcal{P}_{n}$.

The definition of $S$ implies that $S \in \Gamma_{Y}$ and

$$
\{x \in X: S x \neq R x\} \subset \bigcup_{i=-k_{0}}^{k_{0}} T^{i} B\left(\mathcal{P}_{n}\right) .
$$

Therefore, by (3.3) we conclude that $S \in U$.

Acknowledgements. This work was done when the author was visiting the Torun University. I am thankful to the institution for the hospitality and support. Also I would like to thank S. Bezuglyi and J. Kwiatkowski for helpful discussions.

\section{References}

[B-D-K 1] S. Bezuglyi, A. H. Dooley, and J. Kwiatkowski, Topologies on the group of Borel automorphisms of a standard Borel space, Topol. Methods Nonlinear Anal. 27 (2006), 333-385. 
[B-D-K 2] S. Bezuglyi, A. H. Dooley, and J. Kwiatkowski, Topologies on the group of homeomorphisms of a Cantor set, ibid. 27 (2006), 299-331.

[B-D-M] S. Bezuglyi, A. H. Dooley, and K. Medynets, The Rokhlin lemma for homeomorphisms of a Cantor set, Proc. Amer. Math. Soc. 133 (2005), 2957-2964.

[B-K] S. Bezuglyi and J. Kwiatkowski, The topological full group of a Cantor minimal system is dense in the full group, Topol. Methods Nonlinear Anal. 16 (2000), 371-397.

[B-K-M] S. Bezuglyi, J. Kwiatkowski, and K. Medynets, Approximation in ergodic theory, Borel, and Cantor dynamics, in: Contemp. Math. 385, Amer. Math. Soc., 2005, 39-64.

[B-M] S. Bezuglyi and K. Medynets, Smooth automorphisms and path-connectedness in Borel dynamics, Indag. Math. 15 (2004), 453-468.

[C-F-S] I. Cornfeld, S. Fomin and Ya. Sinai, Ergodic Theory, Grundlehren Math. Wiss. 245, Springer, 1982.

[G-P-S] T. Giordano, I. Putnam, and C. Skau, Full groups of Cantor minimal systems, Israel J. Math. 111 (1999), 285-320.

[G-K] E. Glasner and J. King, A zero-one law for dynamical properties, in: Topological Dynamics and Applications (A volume in honor of Robert Ellis), Contemp. Math. 215, Amer. Math. Soc., 1998, 215-242.

[Gl-W] E. Glasner and B. Weiss, The topological Rohlin property and topological entropy, Amer. J. Math. 123 (2001), 1055-1070.

[Hal 1] P. Halmos, Approximation theories for measure preserving transformations, Trans. Amer. Math. Soc. 55 (1944), 1-18.

[Hal 2] - Lectures on Ergodic Theory, Publ. Math. Soc. Japan 3, Math. Soc. Japan, 1956.

[Ke-Ros] A. Kechris and C. Rosendal, Turbulence, amalgamation and generic automorphisms of homogeneous structures, ArXiv:math.LO/0409567 v2, 30 September 2004 .

[Kn-R] B. Knaster et M. Reichbach, Notion d'homogénéité des homéomorphies, Fund. Math. 40 (1953), 180-193.

[K-W] J. Kwiatkowski and M. Wata, Dimension and infinitesimal groups of Cantor minimal systems, Topol. Methods Nonlinear Anal. 23 (2004), 161-202.

[M] K. Medynets, Cantor aperiodic systems and Bratteli diagrams, C. R. Math. Acad. Sci. Paris 342 (2006), 43-46.

[Mil] B. Miller, Density of topological full groups, preprint, 2006.

$[\mathrm{N}]$ M. Nadkarni, Basic Ergodic Theory, 2nd ed., Birkhäuser, 1998.

[Ro] V. A. Rokhlin, Selected topics from the metric theory of dynamical systems, Uspekhi Mat. Nauk 4 (1949), no. 2, 57-128 (in Russian); English transl.: Amer. Math. Soc. Transl. 49 (1966), 171-240.

[W] B. Weiss, Measurable dynamics, in: Contemp. Math. 26, Amer. Math. Soc., 1984, 395-421.

Department of Mathematics

Institute for Low Temperature Physics

47 Lenin ave.

61103 Kharkov, Ukraine

E-mail: medynets@ilt.kharkov.ua

Received 23 August 2005;

in revised form 30 December 2006 